\title{
Automobilsicherheitsgürtel für Schwangere
}

Erklärung des Committee on Medical Aspects of Automotive Safety der American Medical Association

Schwangere unternehmen häufig Autofahrten. Sie besuchen den Arzt, das Krankenhaus; sie fahren zur Kirche und zum Einkaufen und trans-portieren ihre Kinder. Das Risiko eines Zusammenstosses ist für Schwangere das gleiche wie für andere Frauen. Das «Komitee für die medizinische Sicherheit beim Autofahren» empfiehlt seit 1967 alien Insassen von Auto-mobilen Sicherheitsgurte anzulegen; auch Schwangeren wird das empfoh-len. Man meint, dass die Sicherheit für die Schwangeren selbst wie auch für den Fetus erhöht sei, wenn ein Beckengurt getragen würde; es wurde auch das Tragen eines Schultergurtes empfohlen. Es sind anderseits aber auch Verletzungen durch das Tragen von Gürteln mitgeteilt worden. Andere Schutzsysteme für Autoinsassen sind aber noch unvollkommener. Die Bewährung von Sicherheitsgurten sollte also überprüft werden.

Verletzungen durch den Gürtel wurden in 5\% der Unfälle berichtet; allerdings waren davon 90\% oberflächlicher Art. Der Gürtel verhindert zuverlässig das Herausgeschleudertwerden bei Unfällen. Ein Bericht aus Schweden enthielt unter 28000 Zusammenstössen nur 3 tödliche Ausgänge bei Gürtelträgern und nur wenige ernsthafte Verletzungen. Sind diese sehr günstigen Ergebnisse des Schutzes durch Tragen von Sicherheitsgurten auch für schwangere Frauen gültig? Experimentell hat man schwangere Paviane einem Anpralldruck von $20 \mathrm{~g}$ - das entspricht einer plötzlichen Bremsung bei 70 km Stunden-geschwindigkeit - ausgesetzt. Der intrauterine Druck wurde dabei durch das Anlegen eines Schultergürtels nicht vermindert, obgleich die Gefähr-dung des Feten durch Einklemmen des Uterus zwischen Wirbelsäule und Gürtel dabei geringer ist.Eine weitere experimentelleUntersuchung zeigte, dass das Tragen eines Schultergürtels die beim Aufprall entstehende Beu-gung über den Hüftgürtel verhindert und der dabei sonst beobachtete Abfall der Herzfrequenz des schwangeren Tieres vermieden wird. Bei einigen Tieren sah man erhebliche Traumatisierungen, jedoch starb keines der Muttertiere an den direkten Folgen des Aufpralls. 3 von 11 Feten hatten erkennbare Aufprallverletzungen, es starben aber alle 11 Feten.

1 Originaltitel: Automobile Safety Belts During Pregnancy.

242 Committee on Medical Aspects of Autonome Safety

Daran könnten allerdings die experimentellen Manipulationen an den Feten, das Anbringen von

Transducern usw. beteiligt sein.

In einer anderen Untersuchungsreihe (Wayne University) wurden bei schwangeren Pavianen Bremsungen von 40 g vorgenommen, die ohne tödliche Folgen für die Muttertiere und die Feten blieben, selbst bei mehr-fachem Aufprall, wenn anstelle von Gürteln Netze benutzt wurden. Der dabei beobachtete Anstieg des intrauterinen Druckes und der Herzfre-quenz der Muttertiere war dabei der gleiche wie bei den vorher erwähnten Untersuchungen.

Ähnlich wurde in einer weiteren Untersuchung (Wayne University) der Beckengurt mit einem Dreipunkt-Gurt verglichen (Beckengurt-Schulter-fixierung). Bei einer Bremsung mit 23 g starben 50\% der Feten, wenn die Muttertiere einen Beckengurt trugen; es starben nur 8\% der 
Feten, wenn die Muttertiere Schulterfixierung und Beckengurt trugen. Keines der Muttertiere erlitt irgendwelche wesentlichen Verletzungen.

Erfahrungen beim Menschen

Umfangreiche Informationen über schwangere Frauen sind in einer kalifornischen statistischen Untersuchung zusammengestellt, die 441 Auto-mobilinsassen umfasst. Tödlicher Ausgang des Unfalls kam bei Schwangeren ohne Gürtel etwa doppelt so häufig vor wie bei Schwangeren mit Gürtel. Die Sterblichkeit der Feten, einschliesslich derer, die mit ihren Müttern starben, wurde durch Gürteltragen nicht beeinflusst. Auch die Häufigkeit und Schwere von Verletzungen der Mutter waren bei den Gürtelträgerinnen gering. Der Unterschied war wegen der kleinen Fallzahl statistisch nicht signifikant. Signifikant war hingegen der Unterschied bei der Zahl der Verletzten und der tödlich Verunglückten, die herausge-schleudert wurden, gegenüber denen, die im Wagen verblieben. Von 180 Automobilinsassen ohne Gürtel, die herausgeschleudert wurden, starben 33\%; von denen, die im Wagen blieben, $5 \%>$. Schwere Verletzungen gab es unter den Herausgeschleuderten in 72\%, unter den Nicht-Herausge-schleuderten in 15\% > der Fälle. 47\% der herausgeschleuderten Schwangeren hatten tote Feten, von den im Wagen gebliebenen Schwangeren starb nur in 11\% der Fetus. Da für Automobilinsassen ohne Gürtel statistisch signifikante Korrela-tionen zwischen Herausgeschleudertwerden und Tod bestehen, sollten Beckengürtel, die das Herausgeschleudertwerden im allgemeinen verhin-dern, empfohlen werden.

Aus: J. amer. med. Ass. 227: 20-25 (1972); cit. Gynäk. Rdsch. 13: 241-242 (1973) 